\title{
Avaliação in vitro da patogenicidade de fungos predadores de nematóides parasitos de animais domésticos
}

\section{In vitro evaluation of nematophagous fungi patogenicity against nematode of domestic animals}

\author{
Érika Barbosa Neves Graminha',2; Arlete Silveira Maia² ; Jaime Maia dos Santos ${ }^{3}$; \\ Regina Célia Cândido4; Giane Serafim da Silva²,5; Alvimar José da Costa²
}

\begin{abstract}
Resumo: O controle biológico é uma alternativa promissora no controle dos estágios pré-parasitários de helmintos, por meio da ação de fungos nematófagos. Com este propósito, o presente estudo avaliou in vitro a patogenicidade dos fungos Arthrobotrys musiformis e A. conoides sobre larvas infectantes de Haemonchus contortus, Ancylostoma spp. e ovos larvados de Ascaridia galli. Foram utilizados nove grupos: seis tratamentos (G1A e G2A: L3 de H. contortus; G1B e G2B: L3 de Ancylostoma spp.; G1C e G2C: ovos larvados de A. galli, com A. musiformis (G1) e A. conoides (G2), respectivamente) e três testemunhos (G3A, G3B e G3C: larvas de $H$. contortus, Ancylostoma spp. e ovos de $A$. galli, respectivamente, na ausência do fungo). Cada tratamento constou de dez repetições (placas de Petri, de $90 \mathrm{~mm}$ de diâmetro, com média de 120 larvas de $H$. contortus, 200 larvas de Ancylostoma spp. e média de 100 ovos de $A$. galli). Os resultados demostraram que $A$. musiformis predou $66,33 \%$ e $94,44 \%$ das larvas infectantes de Ancylostoma spp. e $H$. contortus, respectivamente. Enquanto que $A$. conoides predou $51,76 \%$ e $89,39 \%$ das mesmas larvas. Os fungos nematófagos avaliados não apresentaram predação sobre ovos larvados de $A$. galli. Portanto, ambos os fungos mostraram-se promissores quanto a sua utilização no biocontrole desses helmintos parasitos de animais domésticos. Palavras-chave: fungos nematófagos, nematóides, controle biológico.
\end{abstract}

\begin{abstract}
Biological control is a promising alternative for the control of pre-parasitic stages through the action of nematophagous fungi's. The present study evaluated in vitro the patogenicity of both Arthrobotrys musiformis and $A$. conoides fungi on "infective larvae (or L3 state larvae)" of Haemonchus contortus, L3 larvae of Ancylostoma spp. and larvated eggs of the nematode Ascaridia galli. Nine groups were formed: six treated groups (G1A and G2A: L3 of $H$. contortus; G1B and G2B: L3 of Ancylostoma spp.; G1C and G2C: larvated eggs of $A$. galli, being that G1 with $A$. musiformis and G2 with $A$. conoides) and three untreated groups (G3A, G3B and G3C: larvae of $H$. contortus, Ancylostoma spp. and eggs of $A$. galli, respectively, without fungi). Each treatment had ten repetitions (ten Petri dishes with an average of 120 larvae of $H$. contortus and 200 larvae of Ancylostoma spp. and, approximately, 100 larvated $A$. galli eggs). The results showed $A$. musiformis plundered $66 \%$ and $94 \%$ of Ancylostoma spp. L3 larvae and $H$. contortus L3 larvae, respectively. While that $A$. conoides plundered $51,7 \%$ and $89,4 \%$ at the same larvae. The nematophagous fungi evaluated have not presented any predation type on $A$. galli larvated eggs. Afterwards, both are promising fungi as to their use for biological control of parasitic helminths of animal hosts.
\end{abstract}

Key words: nematophagous fungi, nematodes, biological control.

\section{Introdução}

O prejuízo causado por endoparasitismo é considerável na produtividade de bovinos, sendo a infecção por nematóides a principal causa de perdas econômicas em todo mundo (HORN e ARTECHE, 1985). Tais perdas estão associadas a infecções gastrintestinais, que provocam efeitos fisiopatológicos nos animais, com conseqüente diminuição no ganho de peso; alterações na condição corporal; efeito depressivo na produção de leite; piora na conversão alimentar; no desempenho reprodutivo dos animais e, ainda, no aumento da mortalidade, principalmente entre animais jovens. Segundo
Bianchin et al. (1993), no Brasil estas taxas de mortalidade variam de $5 \%$ a $10 \%$.

O controle desses parasitas é realizado por meio da aplicação de produtos químicos nos hospedeiros e tem como principais desvantagens: o desenvolvimento de resistência por parte dos parasitas àqueles princípios mais comumente empregados (PRICHARD et al., 1980; SHOOP, 1993), o alto custo de aquisição desses fármacos para o produtor, a possibilidade de ocorrência de resíduos químicos no ambiente (GEORGHIOU e TAYLOR, 1977 ) e nos produtos animais, além das dificuldades técnicas e econômicas para o desenvolvimento de novas

\footnotetext{
${ }^{1}$ Aluna de Doutorado do Curso de Pós-graduação em Microbiologia, Área de Concentração em Microbiologia FCAVJ/UNESP, Campus de Jaboticabal

${ }^{2}$ CPPAR - Centro de Pesquisas em Sanidade Animal, FCAV/UNESP, Campus de Jaboticabal

${ }^{3}$ Depto. de Fitossanidade - FCAV/UNESP, Jaboticabal, Campus de Jaboticabal

${ }^{4}$ Depto. de Análises Clínicas, Toxicológicas e Bromatológicas - FCFRP/USP, Ribeirão Preto - SP

5 UNICASTELO-Universidade Camilo Castelo Branco, Campus VIII
} 
moléculas (CONDER e CAMPBELL, 1995). Alguns pesquisadores, como Alves (1986), Santos e Charles (1995), Charles (1996), Gronvold et al. (1996) afirmaram que o uso do controle biológico pode ser integrado ao uso de produtos químicos, para o controle de pragas e de parasitoses.

Fungos nematófagos compreendem aqueles fungos que atacam nematóides vivos e/ou seus ovos e os utilizam como fonte de nutrientes (MANKAU, 1980). Quatro estratégias principais de ataque são usadas:

1) fungos predadores, nos quais estruturas especiais de captura, adesivas ou não, são formadas sobre o micélio e através delas os nematóides são eficientemente capturados;

2) os endoparasitas, produzindo esporos adesivos ou não, que se fixam na superfície do nematóide ou são ingeridos pelos mesmos;

3) fungos oportunistas ou parasitos de fêmeas sedentárias, de ovos e de cistos;

4) fungos produtores de metabólitos tóxicos.

Os fungos dos dois primeiros grupos capturam nematóides vermiformes. No terceiro grupo, a invasão dos ovos não se dá por meio de estruturas especializadas, tais como armadilhas ou esporos, mas sim pelo crescimento interno de hifas vegetativas, com ou sem a formação do "apressório".

Segundo Barron (1977), esses fungos pertencem à classe dos Deuteromycetes, ou fungos imperfeitos, e incluem-se aqueles que são predadores, endoparasitas ou parasitas de ovos de nematóides.

Ainda de acordo com Alves (1986) e Grønvold et al. (1996), em certos ambientes, a competição entre os microrganismos é tão intensa e severa que praticamente impossibilitaria, em tais situações, a introdução de uma nova espécie fúngica selecionada como agente de controle biológico. Desse modo, o caminho mais sensato, com vistas ao emprego desses microrganismos no controle de nematóides parasitas, seria a seleção de espécies e/ou isolados capazes de atravessarem o trato digestivo dos animais e continuarem viáveis.

Mota et al. (1999) testaram e compararam a atividade predatória dos fungos Arthrobotrys conoides e Monacrosporium thaumasium sobre larvas infectantes de Haemonchus contortus. Os resultados demonstraram que $M$. thaumasium foi estatisticamente mais eficaz na predação de larvas de $H$. contortus $(p>0,01)$. O número de larvas recuperadas dos grupos com $A$. conoides, $M$. thaumasium e controle foram respectivamente: $2.730,690,8.520$. As espécies $A$. conoides e M. thaumasium desenvolvem atividade predatória sobre larvas de $H$. contortus, porém a espécie $M$. thaumasium demonstrou ser mais eficaz nos testes in vitro.

Este estudo teve como objetivo a avaliação da patogenicidade dos fungos predadores Arthrobotrys conoides e Arthrobotrys musiformis em larvas (L3) de Haemonchus contortus (Rudolphi, 1803) de ovinos (Ovis aries), larvas (L3) de espécies da família Ancylostomatidae, prove- niente de cães (Canis familiaris, Linnaeus) e ovos larvados de Ascaridia galli (Shrank, 1788) de galinha (Gallus gallus domesticus).

\section{Material e Métodos}

Os estudos foram conduzidos no Laboratório de Microrganismos Anti-Parasitários do "Centro de Pesquisas em Sanidade Animal" (CPPAR).

Foram três grupos de tratamento para cada nematóide contendo 10 (dez) placas com meio de cultura ágar-água $2 \%(A A)$ :

Grupo Controle - placas com disco de BDA a $2 \%$ (Batata Dextrose Ágar) esterilizado;

Grupo Tratamento 1 - placas com discos de cultura de A. conoides em BDA;

Grupo Tratamento 2 - placas com discos de cultura de A. musiformis em BDA.

Para os testes de patogenicidade foram utilizados dois isolados de fungos oriundos de solos cultivados, obtidos no Laboratório de Nematologia da FCAV/ UNESP - Campus de Jaboticabal, identificados como Arthrobotrys conoides e A. musiformis, segundo as descrições de Haard (1968).

Nematóides de vida livre (NVL), do gênero Panagrellus Thorne, foram criados em meio de aveia (HEINTZ, 1978), para utilização como isca e na multiplicação dos inóculos.

A partir das culturas fúngicas mantidas em tubos de ensaio contendo BDA inclinado, foram removidas porções das bordas das culturas e transferidas para placas de Petri contendo ágar-água (AA) a $2 \%$, deixando-as à temperatura ambiente $\left(25^{\circ} \mathrm{C}- \pm 2^{\circ} \mathrm{C}\right)$ por três dias, em condição de escuro, para o desenvolvimento inicial dos fungos. Foram utilizadas 10 placas de Petri para cada isolado. A essas placas, adicionouse a cada dois dias, durante um período de 15 dias, $1 \mathrm{~mL}$ da suspensão contendo 5000 Panagrellus sp.. Essas placas foram mantidas à temperatura ambiente e em condições de escuro para que o ágar estivesse completamente tomado pelo fungo e ocorresse indução da formação de esporos.

Em cada placa de Petri, onde procedeu-se à multiplicação do inóculo, foram adicionados $2 \mathrm{~mL}$ de água destilada esterilizada e, com auxílio de um pincel, foram removidos conídios da superfície do meio. Esta suspensão foi transferida para frascos esterilizados, tipo penicilina, e armazenados em geladeira a $4^{\circ} \mathrm{C}$. A concentração da suspensão de conídios foi ajustada para $10^{3}$ conídios/mL após contagem em câmara de Newbauer (JANSSON et al., 1985).

\subsection{Cultura de larvas (L3) de Haemonchus contortus e Ancylostoma spp.}

Amostras fecais foram colhidas de um ovino naturalmente infectado por $H$. contortus e de um cão natu- 
ralmente infectado por Ancylostoma spp. e colocadas com vermiculita dentro de copos, até obter-se uma consistência conveniente. A seguir, incubou-se em estufa, tipo B.O.D., a $27^{\circ} \mathrm{C}$ e umidade aproximada de $80 \%$ durante sete dias. Coproculturas prévias efetuadas nas fezes desses animais, segundo a técnica de Robert e O'sullivan (1950), demonstraram que as larvas infectantes presentes nas fezes, eram, em sua totalidade, da espécie $H$. contortus e de Ancylostoma spp..

Findo o período de incubação, o material de cada copo foi colocado em funil de Baermann (NICHOLAS, 1975). Quando as larvas migraram para o fundo dos tubos adaptados aos funis, foram colhidas em suspensão aquosa. A seguir, foram centrifugadas a 1000 rpm por cinco minutos, cinco vezes consecutivas, com água destilada, possibilitando uma diminuição dos contaminantes.

\subsection{Cultura de ovos de Ascaridia galli}

Ovos de $A$. galli foram obtidos a partir de fêmeas retiradas do intestino delgado de aves naturalmente parasitadas. Os úteros das fêmeas foram dissecados e os ovos separados. A seguir, foram tratados com solução de pepsina $1 \%$ para remoção dos restos do útero. A suspensão contendo os ovos foi mantida em um béquer, em repouso, e o sobrenadante foi descartado, e os ovos foram lavados em água destilada. Os ovos em suspensão aquosa foram centrifugados a 1000 rpm por cinco minutos, cinco vezes consecutivas e distribuídos em placas de Petri numeradas, as quais foram deixadas em repouso para continuidade da embriogênese por 15 dias, em estufa tipo B.O.D., a $27^{\circ} \mathrm{C}$ e umidade em torno de $90 \%$, até a obtenção da forma infectante, ou seja, larvas L3.

\subsection{Infecção Experimental de Larvas (L3) de Haemonchus contortus e Ancylostoma sp. e ovos de Ascaridia galli}

Das bordas das culturas fúngicas em placas de Petri, contendo BDA, foram removidos discos de micélio de $1 \mathrm{~cm}$ de diâmetro, transferindo-os para 10 placas de Petri, contendo meio AA (2\%), para cada nematóide. Foram utilizados quatro discos eqüidistantes por placa. A essas placas, após três dias à temperatura de $25^{\circ} \mathrm{C}$ e em condições de escuro, foi adicionado 1,0 $\mathrm{mL}$ da suspensão contendo em média 120 larvas infectantes (L3) de $H$. contortus, 200 larvas infectantes (L3) de Ancylostoma sp. e 1,0 mL da suspensão da última lavagem dos ovos de Ascaridia galli, a qual continha, em média, 100 ovos/mL. Como testemunho, foram utilizados discos de meio BDA sem o fungo. Efetuaram-se dez repetições para cada tratamento e para cada nematóide. As placas foram mantidas no escuro, a $25^{\circ} \mathrm{C}$. Após $24 \mathrm{~h}$, foram observadas diariamente por um período de sete dias, os tratamentos para H. contortus e Ancylostoma sp., avaliando-se a quantidade de nematóides predados, por meio de um estereomicroscópio óptico comum com aumento de 20X. As larvas predadas observadas durante os sete dias foram, então, contadas e retiradas das placas, diariamente. Em relação aos ovos de $A$. galli, após sete dias, foram removidos com ajuda de espátula e colocados sobre lâminas contendo uma gota de azul de Aman (ARAÚJO et al., 1995). Os ovos foram observados em microscópio óptico comum com aumento de 130X, avaliando-se o efeito ovicida que os fungos exerceram sobre os mesmos.

O percentual de larvas e ovos predados foi calculado com a seguinte fórmula, para todos os ensaios:

$\%$ LP $=n^{\circ}$ médio de larvas ou ovos vivos controle $-n^{\circ}$ médio de larvas ou ovos vivos grupo tratado

$\mathrm{n}^{\circ}$ médio de larvas ou ovos vivos controle

\subsection{Estudo do modo de ação e estrutura de captura dos fungos ao microscópio eletrônico de varredura}

Culturas puras dos fungos avaliados foram repicadas para placas de Petri, contendo AA (2\%) e incubadas no escuro, à temperatura de $27^{\circ} \mathrm{C}$ por 48 horas. A seguir, $1 \mathrm{~mL}$ das suspensões concentradas de ovos de $A$. galli e larvas de $H$. contortus e Ancylostoma spp. foram transferidos para as placas e novamente incubados. No dia seguinte, iniciaram-se os exames quanto à captura pelos fungos. Quando se observou espécimens parasitados e/ou predados, procedeu-se à fixação em glutaraldeído a 3\%, em tampão de fosfato de potássio a 0,05 M e pH 7,4 por 72 horas. Em seguida, foram lavados na solução tampão pura, seis vezes consecutivas em um intervalo de 15 minutos e pós-fixado em tetróxido de ósmio a $2 \%$, no mesmo tampão. Então, foram novamente lavados, como no caso anterior, desidratados em uma série gradual de álcool etílico, secos em secador de ponto crítico, utilizando-se $\mathrm{CO}_{2}$, montados nos porta-espécimes, recobertos com $35 \mathrm{~nm}$ de ouro e elétron-micrografados em microscópio eletrônico de varredura JEOL JSM 5410, operando em $15 \mathrm{kV}$ (MAIA e SANTOS, 1997).

\section{Resultados e Discussão}

Observou-se que $A$. musiformis predou um número maior de larvas de $H$. contortus que de Ancylostoma spp. no terceiro dia da avaliação (Figura 1). A máxima predação de Ancylostoma spp. foi obtida no quarto dia de avaliação, tendo havido decréscimo da taxa de predação de ambos nematóides por $A$. musiformis a partir de então. A predação de ambos nematóides por $A$. conoides foi crescente, obtendo-se a máxima predação de Ancylostoma spp. no quarto dia, enquanto que a máxima predação de $H$. contortus só foi obtida no sexto dia (Figura 1). A eficácia de predação de $A$. musiformis sobre larvas de $H$. contortus foi maior 
(94,44\%) que sobre larvas de Ancylostoma spp. $(66,33 \%)$. Efeito similar foi observado com $A$. conoides. De fato, a eficácia de predação desse fungo sobre larvas de $H$. contortus foi de $89,39 \%$, enquanto que, para Ancylostoma spp., foi de apenas 51,76\% (Figura 1).

Aos sete dias de exposição dos ovos aos fungos, não se detectou ação predatória ou parasítica dos fungos, demonstrando, portanto, a ineficácia destes sobre ovos de A. galli.

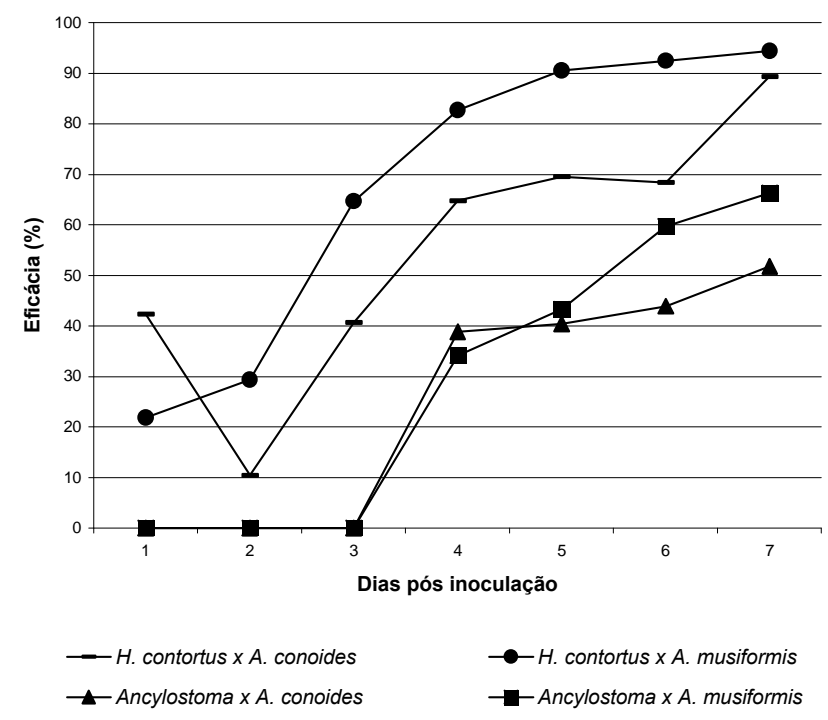

Figura 1 - Percentuais de predação dos fungos nematófagos Arthrobotrys musiformis e A. conoides, sobre larvas infectantes de Haemonchus contortus e Ancylostoma spp., avaliados durante sete dias após a inoculação.

A Figura 2 contém elétron-micrografias de varre-dura ilustrando os conídios e conidióforos de $A$. musiformis (Fig. 2A) e A. conoides (Fig. 2D) e a predação sobre larvas de $H$. contortus (Fig. 2B e 2E, respectivamente) e Ancylostoma spp. (Fig. 2C e 2F, respectivamente).

Figura 2 - Elétron-micrografias de varredura: A) Conídios e conidióforo do fungo Arthrobotrys musiformis. B) Larva (L3) de Haemonchus contortus recém capturada por A. musiformis. C) Larva (L3) de Ancylostoma spp. predada por A. musiformis. D) Conídios e conidióforo do fungo Arthrobotrys conoides. E) Larva (L3) de $H$. contortus predada por A. conoides. F) Larva (L3) de Ancylostoma spp. predada por $A$. conoides. 
A predação in vitro de $94,44 \%$ e $89,39 \%$ das larvas de $H$. contortus, e de $66,33 \%$ e $51,76 \%$ de Ancylostoma spp. por A. musiformis e A. conoides (Figura 1), respectivamente, demonstra que tais fungos nematófagos são potencialmente úteis no controle biológico desses nematóides e que há certa especificidade na relação fungo-nematóide. Com efeito, embora tenha sido colocado maior número médio de Ancylostoma spp. por placa que de $\mathrm{H}$. contortus, ao final do período, de sete dias de exposição dos nematóides aos fungos, houve menor percentual de predação de Ancylostoma spp. que de $H$. contortus. Estes resultados se assemeIham aos de Mendoza-De Gives et al. (1992), que avaliaram a patogenicidade de Arthrobotrys robusta, in vitro, sobre larvas de $\mathrm{H}$. contortus, e encontraram 92,33\% de predação.

A maior eficácia de ambos os fungos sobre $H$. contortus, observada no presente estudo, pelo menos em parte, poderia ser devida ao fato de que ambos os fungos e esse nematóide têm mais chance de interação no campo, já que $H$. contortus é parasito de ovinos e os fungos foram isolados de solo. Ancylostoma spp., por sua vez, foi recuperado de cães de hábito urbano, com chances menores de interação com os fungos em questão e, portanto, com menor suscetibilidade a esses agentes do biocontrole. Embora a eficácia de ambos os fungos tenha sido maior sobre larvas de $\mathrm{H}$. contortus que de Ancylostoma spp., observou-se que a tendência de predação, para ambos os nematóides, foi crescente no decorrer do período experimental. Essas evidências sugerem que pode haver maior probabilidade de se encontrarem agentes do controle biológico de parasitos de cães, com maior patogenicidade e adaptabilidade nos perímetros urbanos, enquanto que agentes do controle biológico de animais criados nas fazendas são mais prováveis de serem encontrados, com essas características, nos solos de pastagens e/ou em bolos fecais.

Além disso, fungos nematófagos que cresçam em fezes frescas dos animais têm maior chance de serem agentes mais eficazes do controle biológico, visto que Nansen et al. (1988) mencionaram que, larvas recém eclodidas, antes de alcançarem o estádio de L3, são mais sujeitas ao ataque desses fungos. As L3 têm duas cutículas que, por si só, constituiriam uma limitação para a ação dos fungos nematófagos.

Os resultados obtidos neste experimento, quanto aos ovos de $A$. galli, são semelhantes aos encontrados por Araújo et al. (1995). Estes autores verificaram que apenas Paecilomyces lilacinus apresentou efeito ovicida sobre ovos larvados de Toxocara canis, enquanto que os fungos do gênero Arthrobotrys ( $A$. robusta e $A$. conoides) apenas exibiram aderência de hifas a casca de ovos.

Segundo Jatala (1986), aparentemente, quando uma forma juvenil é formada dentro do ovo, a atividade parasítica da maioria destes fungos é reduzida. Muitos desses organismos têm atividades quitinolíticas. Assim, como a quitina constitui uma grande porção da casca do ovo, e nas larvas, isto não acontece, os fungos que produzem quitina serão mais específicos na predação dos ovos. Ainda segundo o mesmo autor, a pressão exercida na cutícula do ovo aliada à atividade enzimática, causam ruptura e, subseqüentemente, penetração pelo fungo.

A metodologia utilizada para microscopia eletrônica de varredura (MAIA e SANTOS, 1997) foi muito eficiente para documentação da predação dos nematóides por ambos os fungos. É uma metodologia há muito tempo utilizada e que traz bons resultados. Porém, existem outras metodologias que podem ser utilizadas, principalmente quanto à obtenção das amostras (LYSEK, 1978).

Todos os ensaios apresentaram grande quantidade de bactérias por toda a placa. Porém, segundo Duponnois et al. (1998), algumas bactérias isoladas do conidióforo de $A$. oligospora atuaram como "helpers" na atividade do fungo e por esta razão foram chamadas de NHB (Nematophagous fungus Helper Bacteria), ou seja, bactérias que podem estar envolvidas no sistema de predação, esporulação e patogenicidade deste fungo.

Portanto, é necessário conhecer o papel de todos os organismos envolvidos no biocontrole de nematóides. Segundo Araújo (1999), é essencial que os órgãos financiadores apóiem as pesquisas nessa área, pois muitos aspectos básicos da biologia, da epidemiologia e da interação patógeno - hospedeiro necessitam ser estudados. À indústria caberia o papel muito importante de desenvolver formulações comercialmente viáveis.

Como contribuição subsidiária, os resultados concernentes aos modelos experimentais obtidos com as espécies Ancylostoma spp. e Ascaridia galli poderão ser utilizados em parasitologia humana.

\section{Referências Bibliográficas}

ALVES, S.B. Controle microbiano de insetos. São Paulo: Manole, 1986. 407p.

ARAÚJO, J.V. Controle de nematóides parasitas de bovinos por fungos nematófagos. Cad. Téc. Vet. Zootec., Viçosa, n.30, p.75-88, 1999.

ARAÚJO, M.J.V.; SANTOS, M.A.; FERRAZ, S. Efeito ovicida de fungos nematófagos sobre ovos embrionados de Toxocara canis. Arq. Bras. Med. Vet. Zootec., Belo Horizonte, v.47, n.1, p. 37-42, 1995.

BARRON, G.L. The nematode-destroying fungi. Guelph: Canadian Biological Publications, 1977. 140p.

BIANCHIN, I.;NUNES, S.G.; NASCIMENTO, Y.A. do; CURVO, J.B.E.;COSTA, F.P. Epidemiologia dos nematóides gastrintestinais em bovinos de corte no cerrado e controle estratégico. Campo Grande: EMBRAPA, CNPGC. 1993. 120p. (Circular Técnica, 24).

CHARLES, T.P. Atividade de fungos nematófagos nos estágios pré-parasitários de nematóides tricostrongilídeos. Coronel Pacheco: EMBRAPA, CNPGL, 1996. 21p.

CONDER, G.A.; CAMPBELL, W.C. Chemotherapy of nematode infections of veterinary importance. Adv. Parasitol., v.35, p.58-62, 1995. 
DUPONNOIS, R.; AMADOU, M.B.; MATEILLE, T. Effects of some rhizosphere bacteria for the biocontrol of nematodes of the genus Meloidogyne with Arthrobotrys oligospora. Fundam. Appl. Nematol., Montrouge, v.21, n.2, p.157-163, 1998.

GEORGHIOU, G.P. ; TAYLOR, C.E. Operational in evolution of insecticide resistance. J. Econ. Entomol., College Park, v.70, n.3, p.319-323, 1977.

GRONVOLD, J.; HENRIKSEN, S.A.; LARSEN, M.; NANSEN, P.; WOLSTRUP, J. Biological control: aspects of Biological Control with special reference to arthropods, protozoa and helmints of domesticated animals. Vet. Parasitol., Amsterdam, v.64, p.47-64, 1996.

HAARD, K. Taxonomic studies on the genus Arthrobotrys corda. Mycol., New York, v.6, p.1140-1159, 1968.

HEINTZ, C.E. Assessing the predacity of nematodetrapping fungi in vitro. Mycol., New York, v.70, p.1086-1100, 1978.

HORN, S.C.; ARTECHE, C.C.P. Situação parasitária da pecuária no Brasil. Hora Vet., Porto Alegre v.4, n. 23, p. 12-32, 1985.

JANSSON, H.B.; JEYAPRAKASH, A.; ZUCKERMAN, B.M. Control of root-knot nematodes on tomato by the endoparasitic fungus Meria coniospora. J. Nematol., Jay, v.17, p.327-329, 1985.

JATALA, P. Biological control of plant-parasitic nematodes. Ann. Rev. Phytopathol., v.24, p.453-489, 1986.

LYSEK, H. A scanning electron microscope study of the effect of an ovicidal fungus on the eggs of Ascaris lumbricoides. Parasitol., Cambridge, v.77, 139-141, 1978.

MAIA, A.S. ; SANTOS, J.M. dos. A SEM technique for preparing biological control agents of nematodes in action. Acta Microsc., Caracas, v.6, suppl. B, p.550-551, 1997.

MANKAU, R. Biological control of nematode pests by natural enemies. Ann. Rev. Phytopathol., Palo Alto, v.18, p.415440, 1980.

MENDOZA-DE GIVES, P.; ZAVALETA-MEJIA, E.; QUIROZROMERO, H.; HERRERA-RODRIGUEZ, D.; PERDOMOROLDAN, F. Interaction between the nematode-destroying fungus Arthrobotrys robusta (Hyphomycetales) and Haemonchus contortus infective larvae in vitro. Vet. Parasitol., Amsterdam, v.41, p.101-107, 1992.

MOTA, M.; BEVILAQUA, C.M.L.; ARAUJO, J.V.; ASSIS, L.M. Comparação da atividade predatória de fungos nematófagos das espécies Arthrobotrys conoides e Monacrosporium thaumasium sobre larvas infectantes de Haemonchus contortus. In: SEMINÁRIO BRASILEIRO DE PARASITOLOGIA VETERINÁRIA, 11, 1999, Summary..., Bahia, p.156, 1999.

NANSEN, P., GRÆENVOLD, J., HENRIKSEN, S.A., WOLSTRUP, J. Interactions between the predacious fungus Arthrobotrys oligospora and third-stage larvae of a series of animal-parasitic nematodes. Vet. Parasitol., v.26, p.32937, 1988.

NICHOLAS, W.L. The biology of free-living nematodes. London: Oxford University Press, 1975. 219p.

PRICHARD, R.K.; HALL, C.A.; KELLY, J.D. The problem of anthelmintic resistance in nematodes. Aust. Vet. J., Brunswick, n.56, p.239-249, 1980.
ROBERT, F.H.S. ; O'SULLIVAN, P.J. Methods for egg counts and larval cultures for strongyles infecting tract of cattle. Aust. Agric. Res., Victoria, n.1, p.99-102, 1950.

SANTOS, C.P.; CHARLES, T.P. Efeito da aplicação de conídios de Drechmeria coniospora em cultivo de fezes contendo ovos de Haemonchus contortus. Arq. Bras. Med. Vet. Zootec., Belo Horiznte, v.47, n.2, p.123-128, 1995.

SHOOP, W.L. Ivermectin resistance. Parasitol. Today, Amsterdam, v.9, n.5, p.154-159, 1993. 